\title{
RF CONDITIONING AND TESTING OF FUNDAMENTAL POWER COUPLERS FOR SNS SUPERCONDUCTING CAVITY PRODUCTION*
}

\author{
M. Stirbet", K. M. Wilson, M. Wiseman, J. Henry, M. Drury, G.K. Davis, C. Grenoble, T. Powers \\ and G. Myneni, Jefferson Laboratory, Newport News, VA 23606 \\ I.E. Campisi, Y. Kang, D. Stout, SNS Project, Oak Ridge National Laboratory, TN 37831.
}

\section{Abstract}

The Spallation Neutron Source (SNS) makes use of 33 medium beta $(0.61)$ and 48 high beta $(0.81)$ superconducting cavities. Each cavity is equipped with a fundamental power coupler, which should withstand the full klystron power of $550 \mathrm{~kW}$ in full reflection for the duration of an RF pulse of $1.3 \mathrm{msec}$ at $60 \mathrm{~Hz}$ repetition rate. Before assembly to a superconducting cavity, the vacuum components of the coupler are submitted to acceptance procedures consisting of preliminary quality assessments, cleaning and clean room assembly, vacuum leak checks and baking under vacuum, followed by conditioning and RF high power testing.

Similar acceptance procedures (except clean room assembly and baking) were applied for the airside components of the coupler. All 81 fundamental power couplers for SNS superconducting cavity production have been RF power tested at JLAB Newport News and, beginning in April 2004 at SNS Oak Ridge.

This paper gives details of coupler processing and RF high power-assessed performances.

\section{INTRODUCTION}

The SNS makes use of superconducting RF cavities resonating at $805 \mathrm{MHz}$ in the fundamental $\mathrm{TM}_{010}-\pi$ mode to accelerate $\mathrm{H}^{-}$ions in the main linac from $185 \mathrm{MeV}$ to $1.0 \mathrm{GeV}$, the initial goal of final energy (the energy range can be $840-1300 \mathrm{MeV}$ with the upgrade) [1].

In order to power the cavities, coaxial couplers were chosen. The couplers must be able to withstand at least the peak power delivered by the SNS klystrons (shown in Table 1), $550 \mathrm{~kW}$ for a $1.3-\mathrm{msec}$ pulse length at a repetition rate of 60 pulses per seconds (pps).

Table 1: Coupler requirements.

\begin{tabular}{|c|c|c|}
\hline Parameter & Operation & Processing \\
\hline Impedance & \multicolumn{2}{|c|}{$50 \Omega$} \\
\hline Peak power & $550 \mathrm{~kW}$ & $2.4 \mathrm{MW}$ \\
\hline Pulse length & $1.3 \mathrm{~ms}$ & $1.3 \mathrm{~ms}$ \\
\hline Repetition rate & $60 \mathrm{pps}$ & $60 \mathrm{pps}$ \\
\hline Average power & Max $53 \mathrm{~kW}$ & Kly. limit \\
\hline Bias & $2.5 \mathrm{kV}$ & $\pm 2.5 \mathrm{kV}$ \\
\hline
\end{tabular}

Qualification for cavity assembly involves performing RF high power tests on each fundamental power coupler. This was done using a dedicated test cart using a

\footnotetext{
* Work supported by U.S. DOE contract DE-AC0500R22725

"Mircea.Stirbet@jlab.org
}

connecting waveguide which allows for simultaneous processing of two fundamental power couplers.

\section{PREPARATION OF THE SNS FPCS FOR RF CONDITIONING}

The fundamental power coupler (FPC) consists of a tapered 50-ohm coaxial line with a planar ceramic window (Figure 1). The design is based on the coupler developed for the KEK B-factory superconducting cavities [2] [3]. To adapt that design to the SNS frequency and to the constraints imposed by the cryomodule geometry and assembly procedures, a number of significant design modifications were implemented. On the vacuum side of the assembly, the outer conductor is double walled, helium gas cooled copper-plated stainless steel and the inner conductor is OFE copper. The coaxial alumina window assembly has four instrumentation ports on the vacuum side: two for vacuum gauges, one for an electron pick-up antenna and one for a sapphire optical view port for an arc detector.

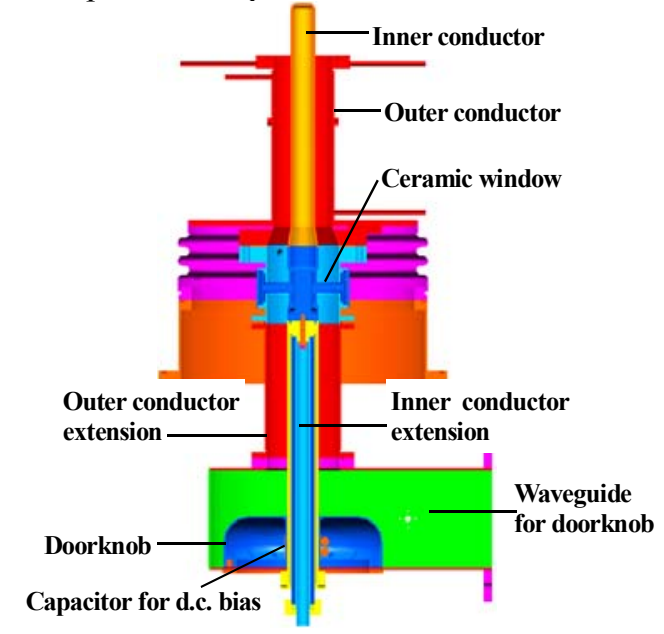

Figure 1: Fundamental Power Coupler for the SNS Project.

On the air side, the outer extension is made from copper-plated stainless steel and the water-cooled inner extension is made of OFE copper. The FPC is matched to a rectangular WR975 waveguide via a waveguide doorknob transition [2][3][5]. Modified Conflat ${ }^{\circledR}$ gaskets are used on the window side to provide good RF contact and at the same time a UHV joint. A capacitor for DC bias is provided between the inner and outer conductors of the coaxial line. 


\section{Test Carts and Connecting Waveguides}

To ensure fluent FPC RF high power testing production style, two test carts and eight custom made UHV connecting waveguides for medium and high beta couplers (dimensioned for $805 \mathrm{MHz}$ resonance with S11 parameter better than $-25 \mathrm{~dB}$ ) have been built [4].

No copper plating was provided on connecting waveguide's surfaces exposed to UHV and RF.

\section{Incoming Inspection}

All components have undergone incoming inspection including full check of dimensions on a coordinate measuring machine; visual examination of the RF exposed surface finish, integrity of vacuum sealing surfaces, followed by cleaning for vacuum leak checks.

The adherence of the copper plating on the outer conductors have been checked by high pressure water rinsing (100 Bar for 30 minutes) and by firing under vacuum for 3 hours at $300^{\circ} \mathrm{C}$. Both methods have proved to efficiently identify copper plating issues (blisters, nonadherent copper).

\section{Component Cleaning and Clean Room Assembly}

Before final assembly, all coupler components were once more carefully cleaned. The outer conductors and all the stainless steel components were cleaned by 15 minutes of immersion in an ultrasonic bath filled with a $10 \%$ solution of Micro-clean ${ }^{\circledR}$ detergent, followed by rinsing with de-ionized water (DI) and drying with dustfree antistatic nitrogen gas. The transfer from the cleaning area to the class 100 clean room was done in double plastic bags filled with dry nitrogen [5].

\section{Mechanical Assembly and Vacuum Tests}

The window assemblies were inserted into the preassembled outer conductors and connecting waveguide.

Aluminum-Magnesium gaskets were used as vacuum seals between the outer conductor and the connecting waveguide ports. The instrumentation ports on the outer conductors were equipped with cold cathode gauges and the window assembly with an electron pick up antenna and one sapphire optical view port. Vacuum leak test were performed on the assembled couplers using a Stanford ${ }^{\circledR}$ RGA. Helium leak detector sensitivity of the system was better than $2 \times 10^{-10}$ Torr $1 / \mathrm{s}$.

\section{Baking}

The assembled FPCs and connecting waveguide were baked on the test stand under vacuum, using a heating box with hot air blower operated via a computer and PLC controller (Figure 2). Our usual baking procedure was applied: ramping up the temperature with a gradient of $10^{\circ} \mathrm{C} / \mathrm{h}$, soaking for 24 hours at $200^{\circ} \mathrm{C}$ then cooling down to room temperature with a controlled gradient of $10^{\circ} \mathrm{C} / \mathrm{h}$. During bake, to avoid oxidization of the RF contacts, an Argon atmosphere was locally provided. Temperature, vacuum and residual gases were continuously monitored and recorded. After bake, at room temperature, vacuum in the system was better than $510^{-10}$ mbar. Use of the heating box with hot air has proved to be very efficient during coupler production.

\section{Low RF Power Measurements}

The air side of the couplers was assembled after baking, using the inner and outer conductor extensions and waveguide/doorknob transitions. Low RF power measurements for $\mathrm{S}$ parameters were performed and used as an estimate for local peak power in the transmission line during RF conditioning and high power testing.

\section{CONDITIONING AND POWER TESTS}

\section{High Power RF Setup}

The $1 \mathrm{MW} 805 \mathrm{MHz}$ pulsed RF power stand at JLAB [6] was used to perform RF conditioning and power tests on all medium beta couplers and a several pairs of high beta couplers. From April 2004, the bake and RF test stands had to be moved, re-commissioned and used at SNS - Oak Ridge (Figure 2) [7].

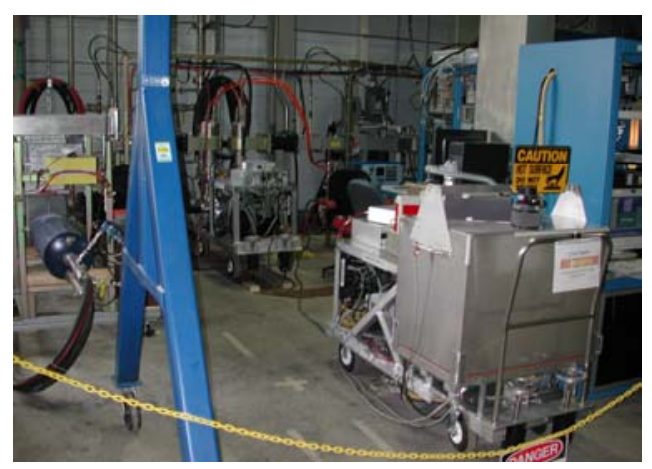

Figure 2: Bake and RF test stands for FPCs processing.

With the two couplers assembled in the test stand, the RF power delivered by the klystron is transferred from the input coupler to the output coupler via the connecting waveguide and dumped into a water load rated for $600 \mathrm{~kW}$ peak power at $8 \%$ duty factor or to a variable short circuit. Three sets of directional couplers (two sets between klystron and test cart and one between the test cart and the terminating load (or variable short circuit) were used to independently monitor the transmitted RF power levels. The RF conditioning was assisted by a fast RF feedback loop, which modulates the RF pulse amplitude as function of FPC vacuum (Figure 3).

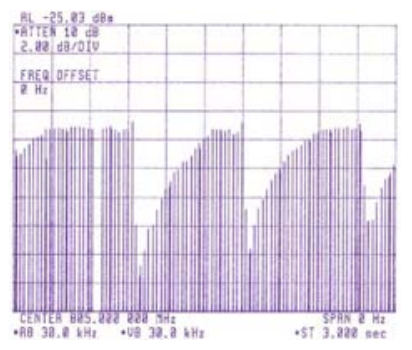

Figure 3: RF pulse amplitude modulation as a function of vacuum. 
A fast interlock system on the vacuum controller's analog output switched RF off if the coupler vacuum was worse than $5 \times 10^{-7}$ mbar. The RF permit was obtained after the vacuum pressure was better than $2 \times 10^{-7}$ mbar. Real time LabView ${ }^{\circledR}$ software provides the operator interface with data acquisition system that allows to adjust and control the RF conditioning or testing parameters [5] [6]. During RF tests, in addition to the vacuum signals, information regarding the electron activity near to the ceramic window is obtained with electrometric instrumentation. A fast photodiode based arc detector was hard interlocked to switch RF off if arcing events occurred on the vacuum side or on the air side (doorknob region) of the coupler.

\section{RF Testing Results}

All 81 SNS FPCs have been qualified for cavity assembly after RF power tests. About 50 hours of RF processing was necessary to assess FPCs RF power performance. During these tests, RF leak measurements were performed and faulty instrumentation identified (vacuum gauges) to be later replaced. After RF processing the FPCs have been transported and stored under dry particulates free nitrogen.

\section{RF Tests on FPCs Vacuum Components}

$\mathrm{RF}$ conditioning as a function of vacuum has been done after baking each pair of couplers. The RF conditioning was started in traveling wave (TW) mode up to $650 \mathrm{~kW}$ pulse amplitude at SNS Oak Ridge (1 MW at JLAB). Usually, after 9-12 hours of RF conditioning the maximum power delivered by the klystron was reached.

$\mathrm{RF}$ conditioning was continued by cycling the pulse amplitude up to the maximum of the RF power delivered by the klystron for at least 12 hours. High RF power tests were then performed by cycling, keeping constant and cycling again the RF amplitude between 10 and $650 \mathrm{~kW}$. The efficiency of d.c. bias in controlling multipacting has been tested while cycling the pulse amplitude up to $650 \mathrm{~kW}$. Last test in TW mode was done with constant pulse amplitude run $(1 \mathrm{msec}, 650 \mathrm{~kW})$ for at least 12 hours (at Oak Ridge this test was done over night).

Tests in standing wave SW mode were performed in steps of forward RF power of $100 \mathrm{~kW}$ up to $600 \mathrm{~kW}$, moving the short circuit over of distance greater than $\lambda / 2$ for at least $30 \mathrm{~min}$ (maximum local peak power along the coaxial line $2.4 \mathrm{MW}$ with $1 \mathrm{~ms}$ pulse duration at $60 \mathrm{~Hz}$ ).

\section{RF Tests on Air Side Components of FPC}

The air side components have been assembled and RF power tested on the room temperature test stand before being assembled on the cryomodule. Two fundamental power couplers have been baked, RF conditioned and then used for testing airside kits in SW mode, ramping the RF in steps of $100 \mathrm{~kW}$ up to $600 \mathrm{~kW} 1 \mathrm{~ms} 60 \mathrm{~Hz}$. During these qualification tests RF leak measurements were performed and d.c. bias efficiency assessed.

\section{SUMMARIES AND CONCLUSIONS}

Testing facilities and procedures established during FPC prototyping at LANL proved to be efficient throughout production. We have demonstrated at JLAB and Oak Ridge that the SNS fundamental power couplers can sustain, on the room temperature test stand, RF power levels in excess of machine specifications. To date, almost all couplers assembled on cavities have been successfully RF powered on the SNS cryomodules at JLAB and SNS [8] [9] and similar results are expected with beam operation in the near future.

\section{ACKNOWLDGEMENTS}

The authors wish to thank the numerous persons who participated in getting activities on SNS fundamental power couplers successfully completed. This work reflects the sustained efforts of working teams from JLAB, LANL (during FPC prototyping) and ORNL.

\section{REFERENCES}

[1] T. E. Mason, "The Spallation Neutron Source: A Powerful Tool for Materials Research," PAC2001, Chicago, IL, June 2001.

[2] Y. Kang et. al., "Electromagnetic Simulations and Properties of the Fundamental Power Couplers for the SNS Superconducting Cavities," PAC2001, http://accelconf.web.cern.ch/AccelConf/p01/PAPERS /MPPH147.PDF

[3] K.M. Wilson et. al., "The Prototype Fundamental Power Coupler for the Spallation Neutron Source Superconducting Cavities: Design and Initial Test Results." 10th Workshop on RF Superconductivity 2001 Tsukuba, Japan 6-11 Sept 2001, http://conference.kek.jp/SRF2001/pdf/PT021.pdf

[4] M. Stirbet et. al., "Processing Test Stand for the Fundamental Power Couplers of the Spallation Neutron Source (SNS) Superconducting Cavities," PAC2001,http://accelconf.web.cern.ch/AccelConf/p0 1/PAPERS/MPPH154.PDF

[5] M. Stirbet et. al., "Testing Procedures and Results of the Prototype Fundamental Power Coupler for the Spallation Neutron Source," PAC2001, http://accelconf.web.cern.ch/AccelConf/p01/PAPERS /MPPH148.PDF

[6] M. Stirbet et. al.,"High Power RF Tests on Fundamental Power Couplers for the SNS Project." EPAC2002, Paris, France, 3-7 Jun 2002. http://accelconf.web.cern.ch/AccelConf/e02/PAPERS /THPDO016.pdf

[7] Y. Kang et al., "High Power RF Test Facility at the SNS", these proceedings - WPAT059

[8] M.A. Drury et al., "Overview of SNS Cryomodule Performance", these proceedings - RPPE060.

[9] I.E. Campisi "Testing of the SNS Superconducting Cavities and Cryomodules", these proceedings ROAC00 